\title{
Étude du clapotis
}

\section{Clapotis}

\author{
PAR G. CHABERT D'HIERES \\ INGÉNIEUR DE RECHERCHES DU G.N.R.S. \\ DÉTACHÉ AU LABORATOIRE DE MÉCANIQUE DES FLUIDES DE L'UNIVERSITÉ DE GRENOBLE
}

\begin{abstract}
Communication présentée à la Société Hydrotechnique de France le 18 juỉn 1959
\end{abstract}
\begin{abstract}
L'auteur donne d'abord diverses définitions du clapotis et les compare entre elles. Il retient celle qui consiste da considérer le clapotis comme un mouvement plan, d'un liquide pesant, avec surface libre, à trajectoires fermées, décrites toutes en même temps, périodique par rapport $\grave{a}$ l'abscisse longitudinale, vertical le long d'un plan vertical fixe. Cette définition conduit da une solution approchée du problème, exacte au $3^{\mathrm{e}}$ ordre près inclusivement par rapport d̀ la cambrure et qui est unique, $\dot{a}$ la transformation d'un groupe près. On montre ensuite que le mouvement ainsi défini est un mouvement irrotationnel.

Cette manière de procéder permet de présenter d'une manière rationnelle les nombreux résultats obtenus par les théoriciens du clapotis el en obtenir des nouveaux, concernant la correclion non linéaire de longueur d'onde, la forme de la surface libre, la pression, etc.

Quelques comparaisons avec les résultats des essais faits en laboratoire complètent l'exposé.
\end{abstract}

\section{Introduction}

On désigne sous le nom de clapotis un type d'oscillation périodique et stationnaire d'un liquide pesant, avec surface libre. Cette définition, trop vague encore, ne peut servir de point de départ à une théorie du phénomène; elle suffit, cependant, pour faire comprendre les difficultés spécifiques de la question.

Il s'agit d'abord d'un mouvement avec surface libre : donc le problème aux limites auquel on ramènera la détermination du mouvement ne sera

\begin{abstract}
Firstly, the author presents the various definitions of clapotis and compares them. He adopts the definition whereby clapotis is considered to be a two-dimensional motion of a heavy liquid with a free surface, all of whose particles take the same time to describe closed trajectories the motion being periodic with respect to the longitudinal axis and taking place in a fixed vertical plane. This definition leads to an approximate solution of the problem which is accurate to the third order of wave steepnesses, and which is inique to the transformation of one group. It is then shown that the motion thas defined is irrotational.

This procedure enables the numerous theoretical results that have been obtained to be presented rationally and to derive new resulls non-linear corrections for wavelength, the shape of the free surface and pressure, etc.

A fetv comparisons are made tilh the results of laboratory tests.
\end{abstract}

pas linéaire. En second lieu, la surface libre étant de forme inconnue a priori, le domaine de définition des solutions (champs des vitesses, par exemple) est a priori aussi inconnu.

Enfin, le clapotis étant un phénomène stationnaire, la forme de la surface libre varie avec le temps; ce fait distingue nettement le clapotis de l'onde progressive. Dans notre cas, il n'existera pas de repère, lié à l'onde, relativement auquel le phénomène soit permanent, et les difficultés qui en résultent ne sont pas banales.

Comme toutes les oscillations périodiques, le 
problème du clapotis est abordé par la méthode du petit paramètre de Poincaré. Le mouvement étant voisin du repos, on suppose que toutes les inconnues sont développables en séries entières par rapport à l'amplitude. L'ensemble des termes en $A^{n}$ de ces séries constitue ce qu'on appelle la $n^{i c m e}$ approximation ou l'approximation du $n^{i i m e}$ ordre. Le théoricien a alors à résoudre deux problèmes : 1) calculer la $n^{\text {icme }}$ approximation en fonction des précédentes, c'est le problème formel; 2) montrer que les séries ainsi obtenues convergent pour A assez petit, c'est le problème d'existence.

Ces questions ont été complètement résolues dans le cas de la houle progressive : citons les Iravaux de Levi-Civita, Struick, Mme Dubreil et Gouyon; ce dernier auteur a obtenu les résultats les plus décisifs, englobant ceux de ses prédécesseurs. Il s'en faut de beaucoup que la théorie du clapotis soit aussi avancée; en particulier, il n'existe -- semble-t-il - aucune démonstration entièrement satisfaisante d'existence de la solution, bien que plusieurs tentatives aient été faites en ce sens. On voit donc que les seuls résultats disponibles au sujet du clapotis sont formels, en ce sens qu'ils sont obtenus à partir des séries dont la convergence reste à démontrer.

La théorie linéaire du clapotis (c'est-à-dire du premier ordre) est classique. Elle ne suffit pas à rendre compte des faits observés. Beaucoup d'auteurs ont alors explicité les approximations des deux premiers ordres en profondeur finie et le calcul a été poussé jusqu'au sixième ordre en profondeur infinie; ainsi le problème formel n'a pas été résolu dans toute sa généralité.

Une des premières difficultés qui subsistent encore semble résider dans le fait qu'il n'existe pas de définition précise du clapotis. R. Miche [1] envisage le clapotis comme résultant, au premier ordre, de la superposition des deux houles linéaires irrotationnelles; il calcule ensuite l'approximation du second ordre, sans établir que sa solution soit la seule possible.

C. Carry [4], Penney et Price [6] postulent a priori que le clapotis est irrotationnel; Carry construit l'approximation au second ordre en superposant deux houles du second ordre de F. Biesel et en complétant le régime par des ondes d'interaction. A. Daubert est allé jusqu'au troisième ordre dans cette voie. Notons que tous les auteurs précités, sauf Penney et Price, paramètrent les inconnues au moyen de coordonnces de repos de Miche. Enfin, J. Sekerz-Zenkovitch part encore de l'approximation du premier ordre, mais en utilisant un système différent de variables indépendantes; de plus, il postule a priori que les approximations d'ordre supérieur possc̀dent une forme détermince.
On voit donc que les méthodes qu'on vient d'analyser présentent un caractère commun : elles ne reposent pas sur une définition précise, intrinsèque du phénomène susceptible de l'isoler dans la vaste classe des mouvements oscillatoires. On se borne au calcul approché du mouvement de façon à vérifier quelques caractères externes du clapotis.

En second lieu, on notera que dans la théorie qui nous occupe, le choix des variables indépendantes est capital. Pour représenter les inconnues (déplacements, vitesses, etc.), il est commode de les paramétrer au moyen des coordonnées d'un point balayant un domaine de forme connue a priori. Or, les artifices utilisés par Levi-Civita, Mme Dubreil et Gouyon, ne s'appliquent pas à l'espèce. C'est pourquoi nous avons eu recours aux coordonnées choisies par R. Miche. Mais ce mode de paramétrage n'est pas entièrement défini a priori -- à supposer qu'il soit légitime -; Kravtchenko et Santon ont beaucoup insisté sur les difficultés qui en résultent. Nous avons pu surmonter cet obstacle et identifier ainsi toutes les solutions approchées précédemment obtenues.

Ce qui précède permet de situer notre contribution personnelle au problème du clapotis.

Nous donnons du clapotis une définition a priori que nous légitimons a posteriori, en montrant que la solution formelle correspondante est unique, grâce, en particulier, aux précisions que nos avons apportées au choix des variables de repos. Mais notre théorie présente à cet égard - il importe de le répéter une fois encore -- deux lacunes : 1) le théorème d'existence reste à démontrer; 2) il n'est pas certain que notre définition soit la seule à convenir aux ondes stationnaires et périodiques par rapporl à une coordonnée spatiale. Nous démontrons ensuite quelques propriétés de notre solution formelle, notamment en ce qui concerne le caractère irrotationnel dı phénomène et les éléments de symétrie qu'il possède.

Ceci fait, nous explicitons les trois premières approximations. Il semble que dans l'état actuel de la technique expérimentale, la précision ainsi atteinte soit largement suffisante dans le cas des profondeurs relatives assez grandes. Il reste, au point de vue formel, deux problèmes essentiels à résoudre : 1) calculer la $n^{i e ̀ m e}$ approximation; 2) étudier théoriquement le phénomène dans le cas des faibles hauteurs relatives.

L'ensemble de nos résultats nous fournit la matière de notre thèse du troisième Cycle d'Hydrodynamique qne nous préparons sous la direction de MM. Kravtchenko et Santon. Nous espérons avoir apporté une contribution utile au difficile problème du clapotis que, pour des raisons mentionnées ci-dessus, nous solnmes loin d'aroir résolu d'une manière salisfaisante. 


\section{Définition du clapotis parfait}

Considérons un canal prismatique à bords verticaux, dont le fond est horizontal et qui est limité dans sa longueur par deux sections droites. Le mouvement étant plan par hypo-

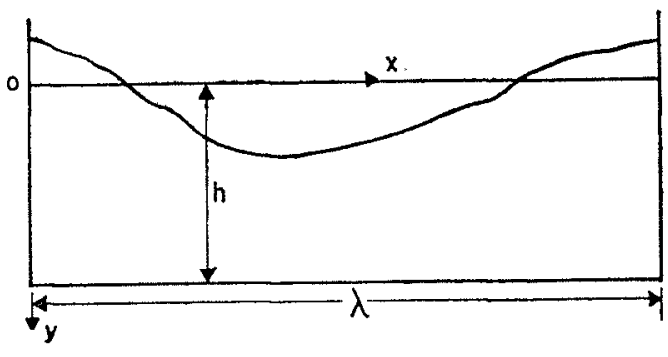

FIG. 1

thèse, nous le rapporterons, comme l'indique la figure, au système de coordonnées rectangulaires $O x O y . \mathrm{O}_{y}$ est orienté suivant la verticale descendante et $\mathrm{O} x$ est situé dans le plan d'eau du repos. On note, $h$ la profondeur du liquide au repos et $\lambda$ la Iongueur du bassin suivant $O x$.

Par définition, le « clapotis parfait » est l'oscillation du liquide contenu dans ce bassin répondant à la condition suivante : la trajectoire de chaque particule fluide est fermée; et toutes les trajectoires sont décrites dans le même temps, 'T, égal à la période du phénomène. Ainsi donc nous commençons par identifier le clapotis à une seiche plane dans un bassin à fond horizontal.

\section{Paramétrage du mouvement}

Pour aborder théoriquement l'étude du clapotis ainsi défini, nous devons maintenant choisir des modes de paramétrage des inconnues; nous serons amenés à faire quelques hypothèses a priori à cette occasion.

a) Pour individualiser chaque molécule c'est-à-dire chaque trajectoire - nous utiliserons les coordonnées moyennes de repos de Miche: $x_{0}, y_{0}$; nous admettons que ce mode de représentation des équations paramétriques soit possible et nous posons :

$$
\begin{aligned}
& x=x_{0}+\mathrm{X}\left(x_{0}, y_{0}, t\right) \\
& y=y_{0}+\mathrm{Y}\left(x_{0}, y_{0} t\right)
\end{aligned}
$$

$u$ et $v$ étant les composantes de la vitesse, on a :

$$
\begin{aligned}
& u=\frac{\partial \mathrm{X}}{\partial t}=u\left(x_{0}, y_{0}, t\right) \\
& v=\frac{\partial \mathrm{Y}}{\partial t}=v\left(x_{0}, y_{0}, l\right)
\end{aligned}
$$

La définition adoptée pour le clapotis entraine la périodicité en $t$ de $\mathrm{X}$ et $\mathrm{Y}$ :

$$
\begin{aligned}
& \mathrm{X}\left(x_{0}, y_{0}, t\right) \equiv \mathrm{X}\left(x_{0}, y_{0}, t+\mathrm{T}\right) \\
& \mathrm{Y}\left(x_{0}, y_{0}, t\right) \equiv \mathrm{Y}\left(x_{0}, y_{0}, t+\mathrm{T}\right)
\end{aligned}
$$

Ce paramétrage du mouvement revient en fait à représenter le domaine occupé par le liquide à l'époque $l$ sur le rectangle fixe $B$, connu $a$ priori.

$$
\begin{aligned}
& 0 \leqslant x_{0} \leqslant \lambda \\
& 0 \leqslant y_{0} \leqslant h
\end{aligned}
$$

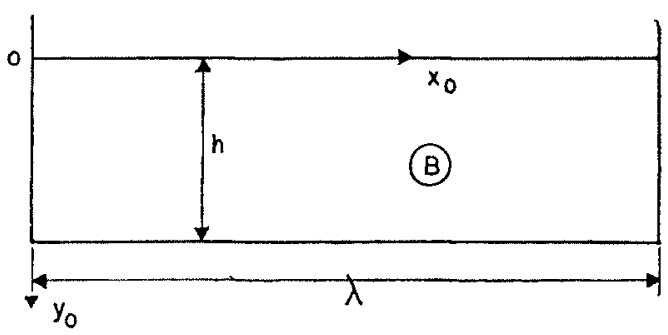

FIG. 2

On voit donc que l'hypothèse faite au début de ce paragraphe permet de paramétrer les trajecloires, c'est-à-dire le mouvement, au moyen de fonctions des variables $x_{0}, y_{0}, t$ balayant un domaine connu a priori; c'est le grand avantage des variables $x_{0} y_{0}$.

b) Nous admettons que toutes les inconnues du problème sont des fonctions analytiques holomorphes à l'origine d'un paramètre A qui caractérise l'amplitude du mouvement; nous préciserons ce point ultérieurement. Nous postulons que les développements des fonctions inconnues suivant les puissances entières de A sont valables pendant une période du mouvement au moins. La périodicité en $t$ garantit alors la convergence pour tout $t$.

\section{Solutions formelles}

Les inconnues $\mathrm{X}\left(x_{0}, y_{0}, t, \mathrm{~A}\right)$ et $\mathrm{Y}\left(x_{0}, y_{1,}, t, \mathrm{~A}\right)$ sont, par hypothèse, développables en séries de 
Taylor en A. Les coefficients de ces séries sont périodiques en $t$, de période T. Comme, d'autre part, $0 \leqslant x_{0} \leqslant \lambda$, on peut, sous réserve d'une hypothèse de régularité, développer ces coefficients en série de Fourier en $x_{0}$, de période $\lambda$. Au point de vue formel, chaque coefficient de $\mathrm{A}^{m}$ de $\mathrm{X}$ et $\mathrm{Y}$ admet donc un développement en série de Fourier double en $x_{0}$ et en $t$. Admettons que la série de Fourier double ainsi obtenue soit absolument convergente. Alors le théorème rels tif à la substitution des séries permet d'écrire :

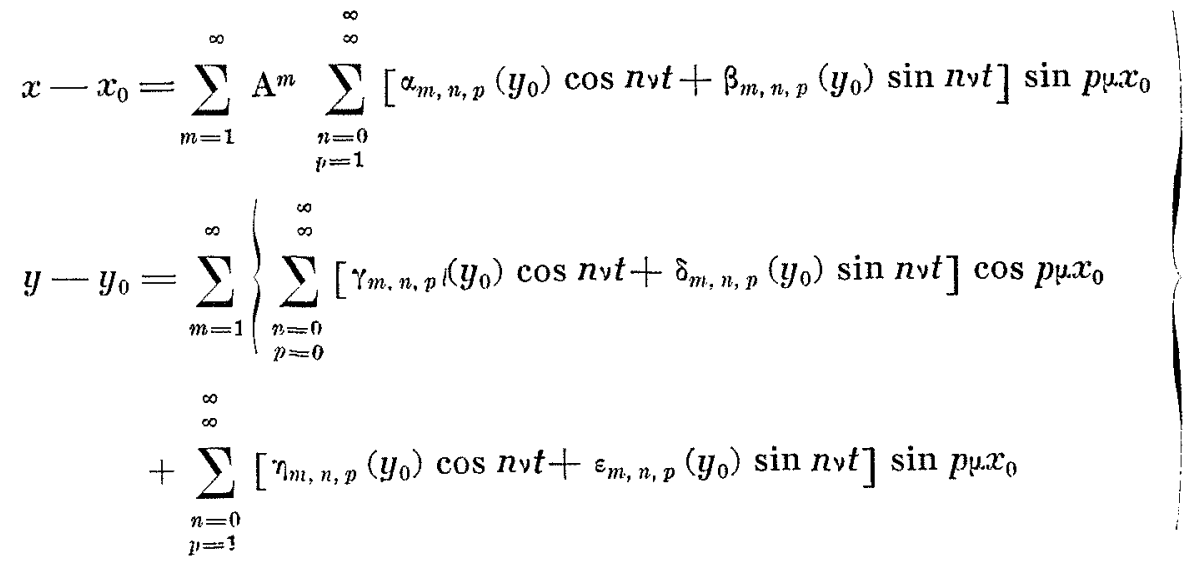

dans lesquelles on a posé :

$$
\nu=2 \pi / \mathrm{T}, \quad \mu=2 \pi / \lambda
$$

$m, n, p$ étant des entiers $\geqslant 0$, variant de 0 à $+\infty$. Ainsi donc, l'hypothèse faite concernant la forme des trajectoires et leur mode de description permet d'éliminer a priori toutes les difficultés qu'entraîne la présence éventuelle des termes séculaires dans les développements de $\mathrm{X}$ et de $\mathrm{Y}$.

Il y a lieu maintenant de faire a priori quelques hypothèses supplémentaires de régularité au sujet des développements formels que l'on vient d'introduire.

Nous avons, en effet, à écrire que ceux-ci vérifient les équations de l'hydrodynamique. A cet effet, nous aurons à les dériver formellement (c'est-à-dire terme à terme) deux fois en $t$ et une fois en $x_{0}$ et $y_{0}$. Or, on sait que ces opérations ne sont légitimes que pour les fonctions assez régulières. Nous admettrons donc que :

1) Les dérivées partielles $\partial^{2} \mathrm{X} / \partial t^{2}, \partial^{2} \mathrm{Y} / \partial l^{2}$ existant, sont «assez » continues en $t$ (par exemple, il suffit qu'elles soient höldériennes, d'exposant supérieur à $1 / 2$ ) pour $0 \leqslant t \leqslant \mathrm{~T}$ et vérifient les conditions :

$$
\begin{aligned}
& \left.\frac{\partial^{2} \mathrm{X}}{\partial t^{2}}\right|_{t=+0}=\left.\frac{\partial^{2} \mathrm{X}}{\partial t^{2}}\right|_{t=\mathrm{T}-0} \\
& \left.\frac{\partial^{2} \mathrm{Y}}{\partial t^{2}}\right|_{t=+0}=\left.\frac{\partial^{2} \mathrm{Y}}{\partial t^{2}}\right|_{t=\mathrm{T}-0}
\end{aligned}
$$

pour toute particule fluide : cela revient à pos- tuler l'existence des accélérations, périodiques en $t$ et continues sur $-\infty \leqslant t \leqslant \infty$ dans tout le rectangle $B$, frontière comprise.

2) Les dérivées :

$$
\frac{\partial \mathrm{X}}{\partial x_{0}}, \quad \frac{\partial \mathrm{X}}{\partial y_{0}}, \quad \frac{\partial \mathrm{Y}}{\partial x_{0}}, \quad \frac{\partial \mathrm{Y}}{\partial y_{0}},
$$

existent, sont continues au sens de. Hölder en $x_{0}$ pour $0 \leqslant x_{0} \leqslant \lambda$ et vérifient les conditions :

$$
\begin{aligned}
& \left.\frac{\partial \mathrm{X}}{\partial x_{0}}\right|_{x_{0}=+0}=\left.\frac{\partial \mathrm{X}}{\partial x_{0}}\right|_{x_{0}=\lambda-0} \\
& \left.\frac{\partial \mathrm{Y}}{\partial x_{0}}\right|_{x_{0}=+0}=\left.\frac{\partial \mathrm{Y}}{\partial x_{0}}\right|_{x_{0}=\lambda-0}
\end{aligned}
$$

pour $-\infty \leqslant \mathrm{t} \leqslant \infty ; 0 \leqslant y_{0} \leqslant h$; autrement dil, les dérivées par rapport à $x_{0}$ des déplacements, définies comme fonctions périodiques de $x_{0}$, doivent, de plus, être continues pour $-\infty \leqslant x_{0} \leqslant \infty$.

3) Les dérivées formelles en $y_{0}$ convergent.

Il est évident que de telles hypothèses restreignent la classe des mouvements que nous considérons; mais elles garantissent la validité d'un raisonnement ultérieur. On verra, de plus, que la solution du problème, construite à partir de ces hypothèses, ne peut être qu'irrotationnelle. On peut en déduire que les fonctions $X$ et $Y$ sont analytiques et holomorphes de $x_{0} y_{0}$ en chaque point de $B$, frontières comprises. En sorte que pour justifier a posteriori la méthode que nous suivons, il suffirait de démontrer la convergence en $A$ de notre processus d'approxima- 
tions successives. Mais ce pas décisif n'est pas encore franchi.

Il sera commode parfois d'utiliser l'écriture :

$$
\begin{aligned}
& x-x_{0}=\mathrm{X}=\Sigma \mathrm{A}^{m} \mathrm{X}_{m}\left(x_{0}, y_{0}, t\right) \\
& y-y_{0}=\mathrm{Y}=\Sigma \mathrm{A}^{m} \mathrm{Y}_{m}\left(x_{0}, y_{0}, t\right)
\end{aligned}
$$

\section{Equations du problème}

Compte tenu de la notation précédente, on peut montrer que $X_{m}$ et $Y_{m}$ vérifient le système linéaire :

$$
\begin{gathered}
\frac{\partial^{3} \mathbf{Y}_{m}}{\partial t^{2} \partial x_{0}}-\frac{\partial^{3} \mathbf{X}_{m}}{\partial t^{2} \partial y_{0}} \\
=-\sum_{r_{s}}\left[\frac{\mathrm{D}\left[\left(\partial^{2} \mathbf{X}_{r} / \partial t^{2}\right), \mathrm{X}_{s}\right]}{\mathrm{D}\left(x_{0}, y_{0}\right)}+\frac{\mathrm{D}\left[\left(\partial^{2} \mathbf{Y}_{r} / \partial t^{2}\right) \mathrm{Y}_{s}\right]}{\mathrm{D}\left(x_{0}, y_{0}\right)}\right] \\
\frac{\partial \mathbf{X}_{m}}{\partial x_{0}}+\frac{\partial \mathbf{Y}_{m}}{\partial y_{0}}=-\sum_{r, s} \frac{\mathrm{D}\left(\mathrm{X}_{r}, \mathbf{Y}_{s}\right)}{\mathrm{D}\left(x_{0}, y_{0}\right)}
\end{gathered}
$$

Toutes les sommes $\underset{r, s}{\Sigma}$ écrites ci-dessus et ciaprès s'étendent aux termes tels que $r+s=m$. Les conditions aux limites à imposer à $\mathrm{X}_{m}$ et $\mathrm{Y}_{m}$ s'écrivent, pour $y_{0}=0$ :

$\frac{\partial^{2} \mathbf{X}_{m}}{\partial t^{2}}--g \frac{\partial^{2} \mathbf{Y}_{m}}{\partial x_{0}}=-\sum_{r, s}\left[\frac{\partial^{2} \mathbf{X}_{r}}{\partial t^{2}} \frac{\partial \mathbf{X}_{s}}{\partial x_{0}}+\frac{\partial^{2} \mathbf{Y}_{r}}{\partial l^{2}} \frac{\partial \mathbf{Y}_{s}}{\partial x_{0}}\right]$

pour $y=h$ :

$$
\mathrm{Y}_{m}=0
$$

Les conditions aux limites sur $x_{0}$ sont déjà écrites dans les développements formels. Celles relatives à $t$ sont automatiquement imposées sur la périodicité.

Pour expliciter la solution, il suffit de substituer dans les équations précédentes les $\mathbf{X}_{m}$ et $\mathbf{Y}_{m}$ par leurs développements de Fourier et d'identifier par rapport aux lignes trigonométriques semblables de $x_{0}$ et $t$. Ón est ainsi ramené à des systèmes différentiels ordinaires qui définissent les couples $\alpha\left(y_{0}\right), \gamma\left(y_{0}\right) ; \beta\left(y_{0}\right), \delta\left(y_{0}\right)$. On démontre en même temps que $\varepsilon\left(y_{0}\right) \equiv \eta\left(y_{0}\right) \equiv 0$, quels que soient les indices $m, n, p$; il en résulte la propriété fondamentale de symétrie du monvement par rapport au plan $x_{0}=\lambda / 2$.

La pression et la surface libre se calculent ensuite très facilement au moyen des développements formels analogues à ceux de $\mathrm{X}$ et $\mathrm{Y}$.

\section{Indéterminations \\ Transformations de groupe}

Dans l'équation (6), les composantes du déplacement sont dérivées deux fois par rapport à $t$. Les termes indépendants de $t$ des développements formels disparaissent, et il ne reste, pour déterminer un couple de coefficients $\alpha_{m}\left(y_{0}\right)$, $\gamma_{m}\left(y_{0}\right)$ ou $\beta_{m}\left(y_{0}\right), \delta_{m}\left(y_{0}\right)$ correspondants qu'une seule équation. Il y a donc ici une indétermination. Heureusement, il ne s'agit là que d'une apparence. Ce qui est en fait indéterminé, c'est le choix des variables. Physiquement, on voit bien que les particules étant toutes identiques, on peut les échanger entre elles sans modifier le phénomène. Cette indétermination a été notée par les différents auteurs qui utilisent les coordonnées moyennes; nous allons, en utilisant les indications de Kravtchenko et Santon, analyser cette difficulté de plus près.

On montre aisément que les équations (6) et (7) sont invariantes par le changement de variables du type :

$$
\begin{aligned}
& x_{0}^{\prime}=x_{0}^{\prime}\left(x_{0} y_{0}\right) \\
& y_{0}^{\prime}=y_{0}^{\prime}\left(x_{0} y_{0}\right) \\
& \frac{\mathrm{D}\left(x_{0}^{\prime}, y_{0}^{\prime}\right)}{\mathrm{D}\left(x_{0}, y_{0}\right)}=1
\end{aligned}
$$

Mais, de plus, les conditions aux limites du problème demeurent invariantes, pourvu que la transformation ponctuelle (10) satisfasse les conditions suivantes : elles doivent conserver les frontières du rectangle (B) :

donc :

$$
\begin{aligned}
& x_{0}^{\prime}=0 \text { lorsque } x_{0}=0 \\
& x_{0}^{\prime}=\lambda \text { lorsque } x_{0}=\lambda \\
& y_{0}^{\prime}=0 \text { lorsque } y_{0}=0 \\
& x_{0}^{\prime}=h \text { lorsque } y_{0}=h
\end{aligned}
$$

L'ensemble de ces transformations forment un groupe $(G)$. En effet, l'ensemble en cause contient la transformation identique et son inverse. De plus, si $\mathscr{G}_{1}$ et $\mathscr{G}_{2}$ sont deux transformations du type (10), (11) et (12), il est évident que la transformation produit :

$$
\mathfrak{G}_{3}=\mathfrak{G}_{1} \times \mathfrak{G}_{2}
$$

appartient encore à l'ensemble.

Ainsi donc, un système de coordonnées moyennes n'est défini qu'à une transformation de $(G)$ près; on montre qu'à chaque ordre, une transformation de ce type permet d'annuler un certain nombre de termes arbitraires.

Toutefois, il subsiste des termes que l'on ne peut annuler par le procédé que l'on vient de décrire. Ces termes, dont les valeurs dans la masse liquide sont arbitraires, sont déterminćs 
à la surface libre; c'est à la présence de ces éléments qu'est dû le phénomène connu sous le nom de surélévation du niveau moyen. Les transformations de $(G)$ permettent, sinon de les annuler, de ramener la solution à une forme canonique.

Il convient de noter que la notion d'amplitude doit également être précisée.

Kravtchenko et Daubert ont montré, dans un article paru dans le $\mathrm{n}^{\circ} 3$, de juillet-août 1957 , de La Houille Blanche, que les équations du problème sont invariantes par une transformation de la forme : $\mathrm{A}=\sum_{m=1}^{\infty} \alpha_{m} \mathrm{~A}^{m}$; d'où il résulte qu'il subsiste un arbitraire dans Je choix de $A$. Nous avons alors défini univoquement ce paramètre d'amplitude comme étant l'amplitude du terme à fréquences fondamentales à la fois en $t$ et en $x$ dans l'expression de la surface libre. Autrement dit, le terme qui a les fréquences fondamentales en $t$ et en $x$ ne devra figurer qu'au premier ordre dans l'équation cartésienne de la surface libre.

Ainsi, quel que soit l'ordre d'approximation, notre procédé conduit à une solution unique, parfaitement déterminée. Il faut faire remarquer un cas particulier qui semble nouveau (mais qui, en fait, provient de notre définition).

Si on se donne la longueur $\lambda$ du bassin et le mode de vibration $\lambda / p$ ( $p$ entier), on en déduit la période $\mathrm{T}(\lambda / p)$ correspondante. Dans le cas où :

$$
\mathrm{T}\left(\frac{\lambda}{p}\right)=\frac{\mathrm{T}(\lambda)}{n} \quad n \text { entier }
$$

l'harmonique $p$ en $x$ aura une fréquence telle qu'il sera également l'harmonique $n$ en $t$. S'il en est ainsi, le phénomène sera encore périodique en $t$ et répondra à notre définition du clapotis. On peut montrer que cetle circonstance se produit pour une suite discrète de valeurs de la profondeur relative $h / \lambda$ et qu'à chaque valeur de cette suite correspond au moins un couple de valeurs entières $n$ et $p$ répondant à la relation (13) quelle que soit l'amplitude (pourvu qu'elle reste petite) des composantes correspondantes. Notons que la suite des valeurs de $h / \lambda$ est alors fonction des amplitudes de ces composantes à cause des termes correctifs de la longueur d'onde qui proviennent des ordres supérieurs au premier.

\section{Le clapotis à trajectoires fermées est irrotationnel}

Un des avantages de notre méthode consiste à déterminer le mouvement par les équations des trajectoires, sans avoir besoin d'une hypothèse a priori concernant le vecleur tourbillon, qui est ainsi parfaitement déterminé a posteriori. Le calcul montre alors que ce tourbillon est nul à tous les ordres. Cette propriété est la conséquence de l'hypothèse de la fermeture des trajectoires (Cf. [9]).

Sekerz-Zenkovitch [7] a énoncé la propriélé précédente dans le cas du clapotis, mais sans en publier de démonstration explicite. Cependant le principe de sa méthode est intéressant: cet auteur affirme que le champ des vitesses s'annule à un instant; l'application du théorème de Lagrange établit alors l'existence du potentiel des vitesses pour tout $t$. Lorsqu'il n'existe pas de solutions que nous avons appelées parasites, on vérifie aisément sur nos formules que le champ des vitesses s'annule effectivement à un instant, ce qui justifie la mise en ruvre du raisonnement de Sekerz-Zenkovitch. Si, au contraire, les termes parasites existent, le champ des vitesses ne s'annule à aucun instant; la dé. monstration de Sekerz-Zenkovitch est alors en défaut. Dans ce cas, il faut procéder directement. On porte les expressions des déplacements dans la formule :

$$
\frac{\mathrm{D}[(\partial x / \partial t), x]}{\mathrm{D}\left(x_{0}, y_{0}\right)}+\frac{\mathrm{D}[(\partial y / \partial t), y]}{\mathrm{D}\left(x_{0}, y_{0}\right)}=\zeta
$$

qui donne le tourbillon $\zeta$ et on vérifie qu'il est nul aux premiers ordres.

Un raisonnement de récurrence analogue à celui de la première démonstration permet d'étendre ce résultat à tous les ordres.

\section{Extension de la définition du clapotis} à un phénomène qui intéresse toute la bande

$$
\left(-\infty<x_{0}<+\infty\right),\left(0<y_{0}<h\right)
$$

Nous n'avions, nous le répétons, étudié le clapotis que dans un bassin dont la longueur était réduite à $\lambda$. Comme la solution admet un potentiel des vitesses, nous pouvons prolonger analytiquement ce dernier par symétrie à travers les parois et cela autant de fois que l'on veut. On obtient ainsi une oscillation du liquide dans un canal s'étendant de $-\infty$ à $-\infty$. Cette oscillation est périodique en $x_{0}$, de période $\lambda$.

\section{Quelques résultats}

Les propriétés qui précèdent sont valables quel que soit l'ordre de lapproximation. Pour achever de résoudre le problème formel, il faut expliciter l'approximation d'ordre $n$. Nous n'avons pas réussi à traiter ce problème et nous nous sommes contenté de calculer les approximations 
des trois premiers ordres. Nous renvoyons à nos notes aux comptes rendus de l'Académie des Sciences. Nous signalons que le calcul de l'approximation d'ordre 3 a été fait en collaboration avec M. Carry.

\section{IJONGUEUR D'ONDE :}

On sait qu'une très bonne approximation de la longueur d'onde est donnée, en fonction de la période, par la formule d'Airy :

$$
\lambda_{0}=\frac{g^{2} \mathbf{T}^{2}}{2 \pi} \text { th } \frac{2 \pi h}{\lambda_{0}}
$$

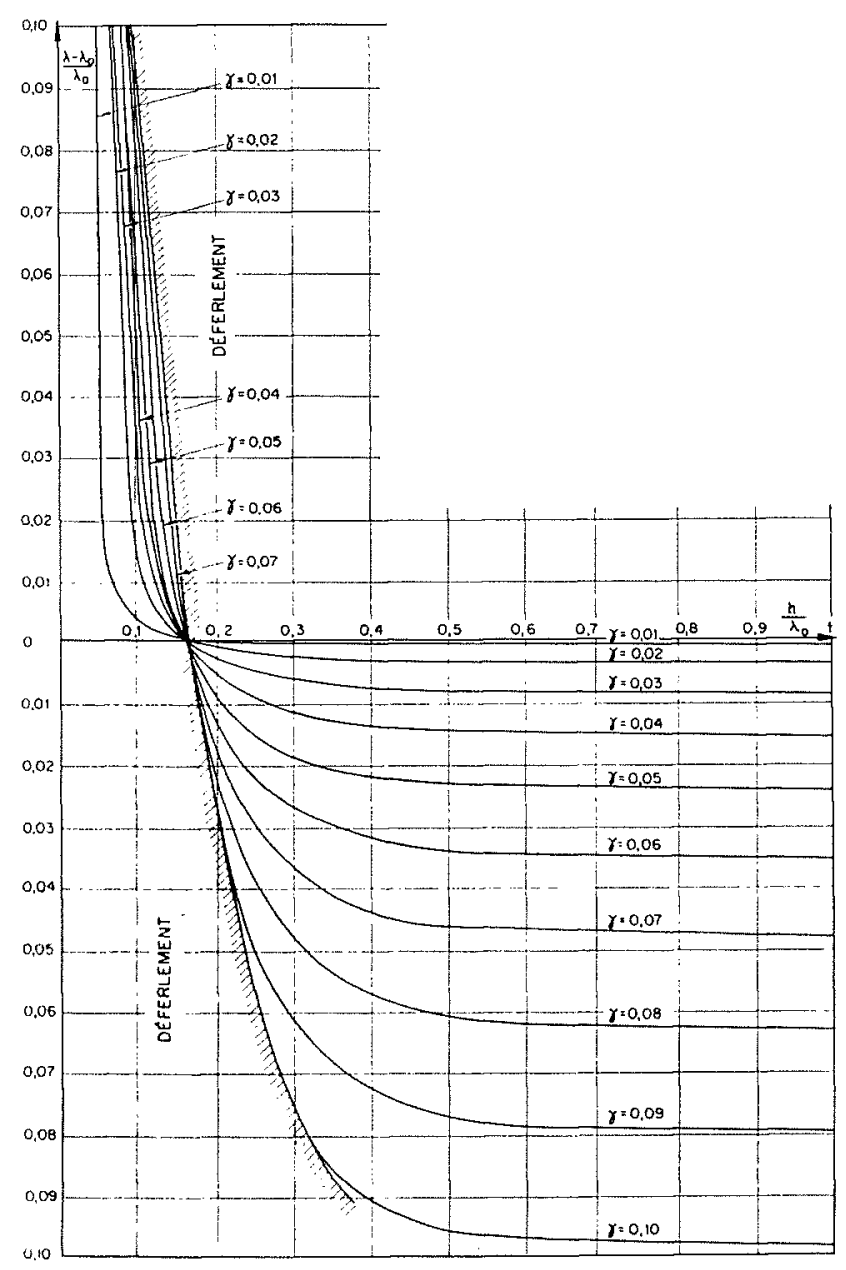

Fig. 3. - Clapotis parfait. Correction de longueur d'onde.

$\lambda_{0}=$ Longueur d'onde d'Airy,

$\lambda$. Longueur d'onde corrigée,

$0_{0}=\operatorname{coth} 2 \pi h / \lambda_{0}$,

$\mathrm{A}=\frac{1}{4}$ Amplitude du fondamental en $t$ et en $x$,

$h=$ Profondeur,

$\gamma=2 \mathrm{~A} / \lambda$,

$\frac{\lambda-\lambda_{0}}{\lambda_{0}}=\frac{-\gamma^{2} \pi^{2}}{80_{0}}-\frac{9 \theta_{0}{ }^{6}+12 \theta_{0}{ }^{4}+3 \theta_{0}{ }^{2}+2}{0_{0}+\mu_{0} / l\left(\theta_{0}{ }^{2}-1\right)}$
Toutefois, le calcul de l'approximation du troisième ordre conduit à une valeur légèrement différente de la longueur d'onde.

La correction à la formule d'Airy peut se mettre sous la forme :

$$
\frac{\lambda-\lambda_{0}}{\lambda_{0}}=\frac{-A^{2} \pi^{2}}{2 \lambda_{0}{ }^{2} \theta_{0}}=\frac{9 \theta_{0}{ }^{6}+12 \theta_{0}{ }^{4}+30_{0}{ }^{2}+2}{\theta_{0}+\mu_{0} h\left(\theta_{0}{ }^{2}-1\right)}
$$

dans laquelle $\lambda_{0}$ est la longueur d'onde d'Airy.

$\lambda$ est la longueur d'onde corrigée.

$$
0_{0}=\cot h\left(2 \pi h / \lambda_{0}\right)
$$

Ainsi donc, $\lambda$ dépend au second ordre de l'amplitude. Sekerz Zenkovitch avait déjà donné une correction similaire. Notons que le polynome du numérateur s'annule une fois pour $0_{0}$ compris dans l'intervalle $\left(1<\theta_{0}<\infty\right)$. Cette racine correspond à la valeur $h / \lambda_{0}=0,1684$ de la profondeur relative. En ce point, la longueur d'onde est, quelle que soit l'amplitude, égale à la longueur d'onde d'Airy, au troisième ordre près, tout au moins. Il est à remarquer qu'aux grandes profondeurs relatives, la longueur d'onde est plus courte que ne l'indique la formule d'Airy. C'est là une propriété particulière au clapotis. La correction de longueur d'onde pour la houle pure irrotationnelle par exenuple ne change pas de signe avec $0_{0}$ et reste positive. La figure 3 donne la valeur de cette correction en fonction de la profondeur relative pour différentes valeurs de la cambrure.

\section{SURFACE LIBRE :}

L’équation cartésienne de la surface libre s'ocrit :

$y=A \cos \mu x \sin \nu t+B_{1} \cos 2 \mu_{x} x+B_{2} \cos 2 \mu x \cos 2$, $+\mathrm{C}_{1} \cos 3 u x \sin v t+\mathrm{C}_{2} \cos \mu x \sin 3 v t+\mathrm{C}_{3} \cos 3 \mu x \sin 3 v t$

Ơn trouvera dans une de nos noles aux comptes rendus de l'Académie des Sciences (t. 245, séance du 21 octobre 1957), les valeurs des coefficients $B_{1}, B_{2}, C_{1}, C_{2}, C_{3}$, dont les représentations graphiques, pour deux valeurs de la cambrure de la houle composante $(0,03$ et 0,07$)$, sont données par les figures 4 et 5 .

\section{Pression :}

L'expression de la pression aux trois premiers ordres d'approximation a été explicitée par C. Carry et noi-même (Cf. [9]). La figure 6 montre l'importance relative des différents termes de la pression au fond du canal et au droit d'un ventre pour une cambrure de la houle composante égale à 0,07 .

P. Jolas a appliqué cette formule aux résultats expérimentaux sur un clapotis parfait, publiés par Kouznielsov. Il note une nette amélio- 

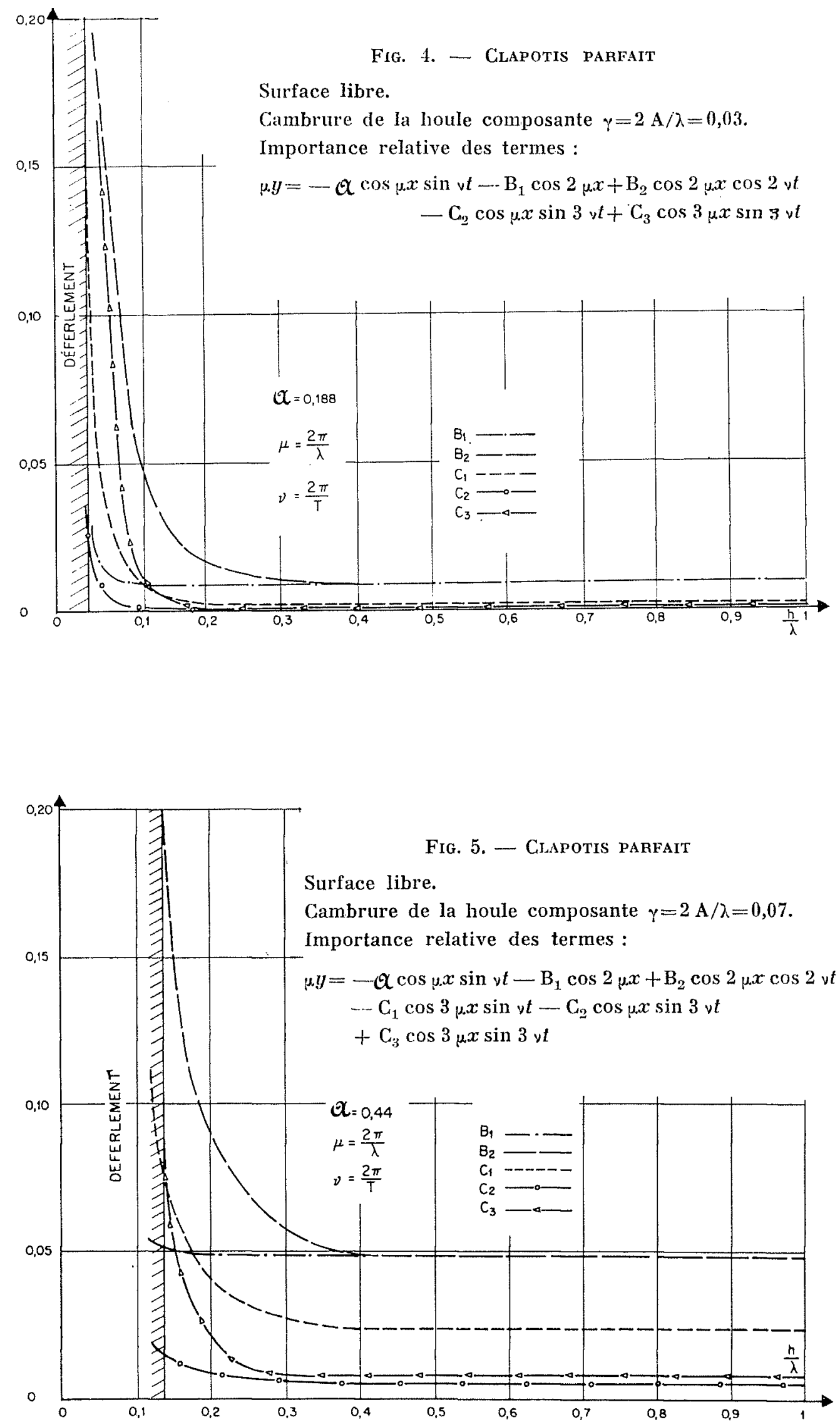

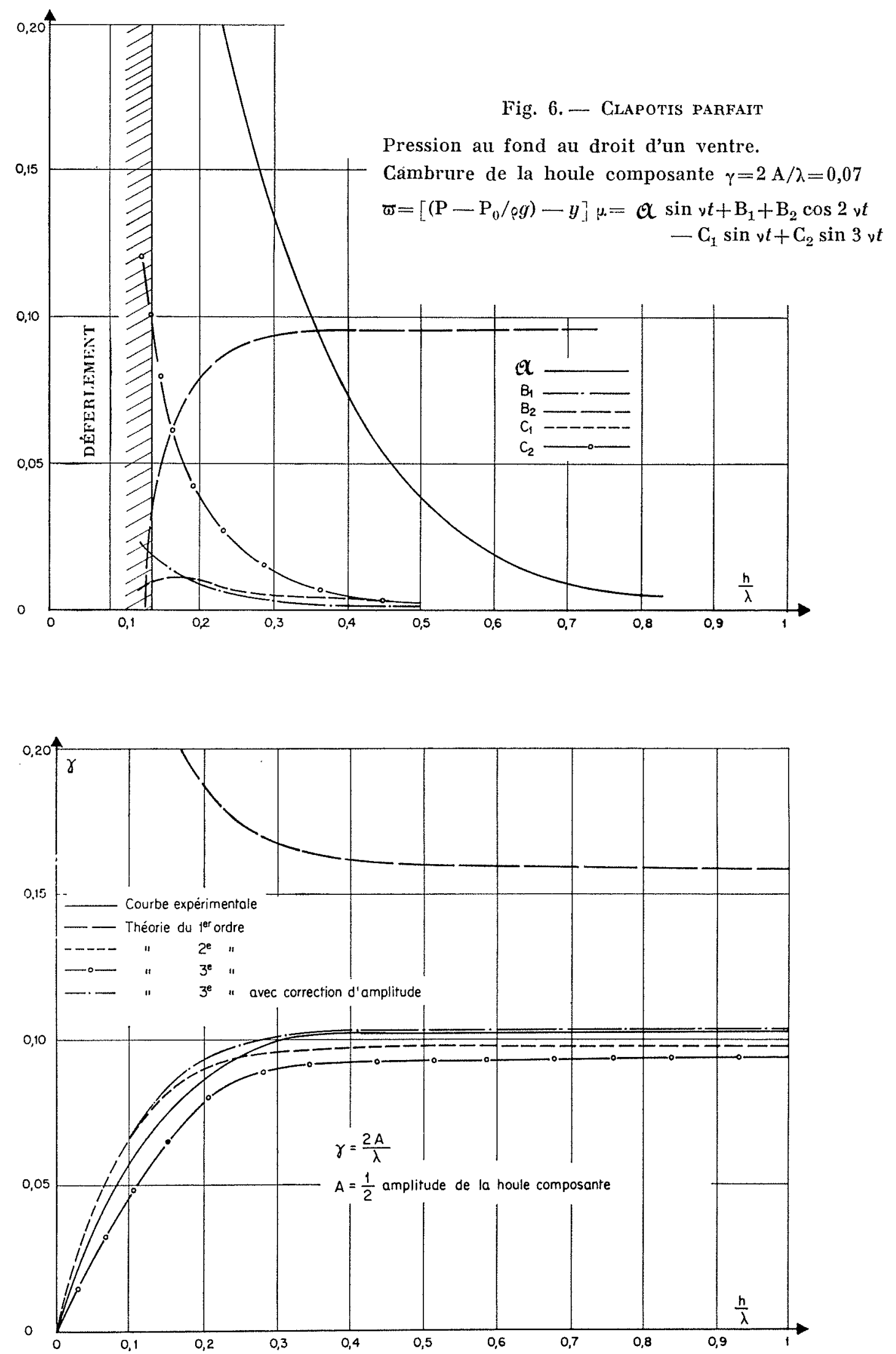

Fig. 7. - Déferlement dU Clapotis 
ration apportée par la prise en compte des termes du troisieme ordre.

\section{DÉFERLEMENT :}

On peut donner une interprétation du déferlement qui semble très bien traduire la réalité physique. Considérons une particule fluide de la surface libre, au point et à l'instant où la cote maxima est atteinte. La vilesse de cette particule est alors nulle. Nous dirons qu'il y a déferlement lorsque l'accélération vers le bas de cette particule est supérieure à $g$. Dans ce cas, une simple application du théorème de Bernoulli généralisé montre qu'en se déplaçant sur une verticale descendante, la pression commencerait à décroître, puis augmenterait à nouveau. La pression en un point situé sous la surface libre, serait inférieure à la pression atmosphérique. Il y aurait en quelque sorte traction des couches profondes sur la surface libre, ce qui semble impossible, d'où le déferlement. La figure 7 montre les limites ainsi obtenues par le calcul aux différents ordres d'approximation, en comparaison avec une courbe expérimentale obtenue par le Laboratoire Dauphinois d'Hydraulique. Une correction d'amplitude a dû être faite, car la valeur de l'amplitude mesurée dans un canal à houle diffère évidemment du double de l'amplitude de la houle composante, à cause des termes du troisième ordre. On constate une très bonne concordance avec l'expérience pour les profondeurs relatives assez grandes (une confrontation analogue a été faite avec le même succès pour la houle).

Notons, pour conclure maintenant, une particularité commune aux houles progressives (rotationnelles ou non) et au clapotis. Les coefficients constants de $\mathrm{A}^{m}$ des développements augmentent indéfiniment avec $\lambda / h$. Cela entraîne que le rayon de convergence en $\mathrm{A}$ des développements en cause doit tendre vers zéro avec la profondeur relative $h / \lambda$. Au point de vue physique, cela veut dire que la houle progressive et le clapotis ne peuvent subsister, pour une cambrure donnée, que si la profondeur relative $h / \lambda$. est assez grande. Ceci explique la discordance entre les courbes théoriques et expérimentales du déferlement pour les faibles valeurs de $h / \lambda$.

\section{BIBLIOGRAPHIE}

[1] Miche (R.). Annales des Ponts et Chrussées, t. 114, 1944, p. 25-61.

[2] Kravtchenko (J.) et Daubert (A.). La Houille Blanche, $\mathrm{n}^{\circ} 3,1957$.

[3] Breser (F.). La Houille Blanche, n*3, 1952, p. 372376.

[4] Carry (C.). La Houille Blanche, $\mathrm{n}^{\circ} 4,1953$, p. 482494.

[5] Gouyon (R.). Thèse (à paraître).

[6] Penney (W. G.) et Price (F. G.). Phil. Trans, Roy. Soc. London, 244, série A, 1951-1952, p. 254-284.
[7] Sererz-Zenkovic (Ja, I.). Ivestia de l'Académie des Sciences de l'U.R.S.S., Série Géographie Géophysique, 15,1951 , p. 57-73.

[8] Rundanen (L.). Bulletin of the Division of Hydranlics, at the Royal Institute of Technology, Stockholm, $\mathrm{n}^{0} 54,1958$.

[9] Chabert d'Hières (G.). C.R.A.S., t. 244, p. 2474-2476; t. 244 , p. $2573-2575$; t. 245 , p. $1377-1379$; t. 246 , p. $1803-1806$.

[10] Jol.as (P.). C.R.A.S., t. 248, pp. 1605-1607.

[11] Kravtchenko (J.) et Santon (L.). Congrès de l'A.I.R.H., Lisbonne, août 1957.

D I S C U S S I O N

président : M. Kravtchenko

M. le Président remercie M. Chabent d'Hiènes et ouvre la discussion.

M. Larras souligne le très gros intérêt des calculs de M. Chabert D'Hrènes qui sont les premiers à traiter directement le problème de l'agitation des eaux devant un mur vertical, alors qu'on n'avait traité jusqu'ici que celui du croisement de deux houles se propageant en sens inverse l'une de l'autre, problème équivalent au précédent en théorie linéaire pour de faibles amplitudes, mais dont rien ne permettait de prouver jusqu'ici qu'il différait peu du précédent pour de grandes amplitudes avec un haut degré de précision. Le mémoire de M. Chabert D'Hières mérite donc largement des félicitations, voire une récompense.
M. LaRras profite de l'occasion pour souligner l'importance des définitions dans les problèmes de houle ou de clapotis, mais aussi la concordance surprenante des résultats obtenus à partir de définitions différentes. Toute définition introduit cependant des restrictions, et M. Larras indique qu'on n'a pas encore traité à sa connaissance le problème du clapotis à trajectoires non fermées, ni celui des limites exactes de convergence des séries utilisées dans les solutions quasi rigoureuses des problèmes de houle et de clapotis d'amplitude finie.

M. Chabert D'Hrères donne les précisions suivantes au sujet de la forme des trajectoires dans le clapotis. Si l'on caractérise le clapotis par la périodicité des vitesses en coordonnées d'Euler, on ne fait aucune hypothèse sur 
la fermeture des trajectoires. Mais on peul démontrer alors que les fonctions représentatrices des déplacements des particules sont périodiques. Les trajectoires sont donc fermées. Les raisonnements qui conduisent à ces vésultats sont fondés sur l'hypothèse de la validité des developpements que l'on utilise. De plus, ces développements doivent être valables pendant un intervalle do temps au moins égal à une période.

En ce qui concerne la convergence des séries, M. le Président signale qu'à Grenoble, toute une équipe de mathématiciens étudie la question et on espère avoir un résultat prochain susceptible de donner lieu à une communication aux séances du Comité Technique de la S.H.F.

Dans la thèse de M. Gouyon, le problème de la convergence a été partiellement résolu pour la houle : en profondeur infinie, les développements formés pour la houle irrotationnelle convergent pour une cambrure égale a $1 / 2000$; celte limite de validité est trop faible pour intéresser le technicien, mais elle peut être aisément améliorée. La méthode au reste se généralise au cas de la profondeur finie et de la houle tourbillonnaire.

On peut signaler également les travaux de M. Jolas sur le contrôle expérimental des formules de M. Chabent D'Hik̀res au moyen des résultats des essais faits pour Kouznetsoff : la définition correcte de l'amplitude améliore singulierement la concordance entre les formules de M. Miche et les résultats de ces observations; les termes du $3^{\circ}$ ordre améliorent encore cette concordance. Une des grandes causes de divergence entre les formules mathématiques et la réalité est simplement la divergence des séries pour des faibles profondeurs : la théorie des mouvements périodiques des eaux en faible profondeur est encore à faire.

\title{
NOTRE FRONTISPICE
}

\author{
(Cf. page 102)
}

\section{HAGTN $(1797-1884)$}

Hagen est de ces savants malchanceux dont le travaux ou les découvertes tardent parfois à obtenir tout le crédit ou la notoriété qu'ils méritent: si, en matière de régime d'écoulement, Hiegen ne parvint pas à établir un paranètre de similitude général tenant compte de la viscosité, il reste évident qu'il précéda Reynolds dans plusieurs découvertes habituellement attribuées à ce dernier.

Gothilf Heinrich Ludwig Hagen naquit à Königsberg le 3 mar Gotthilf Heinrich Ludwig Hagen naquit a Konigsberg le 3 mar 1797 et fit auprès de l'Université de cette ville des études de mathematiques et d'astronomie, en mêne temps qu'il nouait des
rapports étroits avec le mathématicien Bessel.

Une randonnée pédestre d'un an et demi à travers l'Europe centrale lui permit d'y voir et étudier les constructions hydrauli. ques; il commença ensuite à Berlin une carrière remarquable en la triple qualité d'ingénieur de l'Etat, de professeur de constructions hydrauliques it l'Académic d'Architecture, et d'auteur.

Dans le domaine de l'Hydraulique, il s'intéressa particullièrement au problème des résistances en conduites et en canaux découverts. Parmi ses nombrenx ouvrages, il convient de citer : Uebe die Bezoegung des Wassers in engen cylindrischen Röhron (1839), dans lequel on rencontre la prenière expression empirique d'une loi mettant en jeu la viscosité, et la première mention de l'existence de deux modes d'écoulement; Einfluss de Temperatur auf die Bewegung des Wassers in Röhren (1854) Grundziige der Wahrscheinlichkeitsrechnung (1857); Unterst chungen über die gleich förmigc Bereegung des Wassers (1876), Veber dic Ausdehnung des Wassers unter verschiedenet. Wärmegraden (1855) et surtout son cuvre magistrale Handbuch der Wasserbankunst, en trois volumes (1841-1865).

Hagen mourut à Berlin le 3 février 1884. Il était membre de l'Académie des Sciences de Berlin depuis 1842 .
$H A G E N(1797.188+)$ Hagen was one of those unfortunate people whose work some.
times has to wat a long time before it gets all the credit and recoguition it descrves. Although Hagen did not succeed in recogution it deserves. Although Hagen did not succed in
deriving a gencral similitude parameter based on viscosity, it cannot be denicd that he made many of the discoveries wsully attributed to Reynolds.

Gotthilf Heinrich Ludzig Hagen was born on March 3 rd 1797 in Konigsberg and read Mathematics and Astronomy at this in Kongsberg and read Mathematics and Astronomy at this tchon's whiversity. Ditring that period

A roalking tour in Central Europe lasting one and a half years enabled him to see and study the hydraulic structures in that part of the world. He then went to Berlint where he started a rematkable triplc carcer as state engineer, Professor of hydranStructures and anthor.

His main interest in hydrattics was flow resistance in pipes and chen channels. Among his many publications the follozing ar particularly worth mentioning: Ueber die Bewegung des Wasser in engen cylindrischen Röhen (1839), in which we meat the first empirical form of a law involving viscosity and the firs mention of the existence of two modes of flow; Einfluss de Temperatur auf die Bewegung des Wassers in Rören (1854) Grundzäge der Wahrscheinlichkeitsrechnung (1887): Unterstchungen über die gleichförmige Bewegung des Wassers (1876) Ueber dic Ausdehnung des Wassers unter verschiedenen Wärme. graden (1855) and his outstanding Handhuch der Wasserbankinst in three volumes (18+1-1865).

ITagen died in Berlin on Febrnary 3rd 1884. He had been " member of the Berlin Academy of Sciences since 18.42 